\title{
Surgical synovectomy and experimental immune synovitis in the rabbit knee joint
}

\author{
H. L. F. CURREY \\ The London Hospital Medical College, London, E.1
}

AND C. J. MOORE AND A. I. D. PRENTICE

Notley Hospital, Braintree, Essex

Surgical synovectomy is used regularly in the treatment of rheumatoid arthritis, particularly in the knee joint. Pain is often relieved by this operation, but the long-term effects upon the progress of the disease in the operated joint are unknown. It is proving extremely difficult to perform the type of controlled clinical trial needed to determine the long-term results.

Meanwhile, without a better understanding of the pathogenesis of rheumatoid arthritis, the rationale for this operation remains a matter for speculation. It is not known whether any benefit results from excision of inflammatory tissue, removal of antigen, alteration of blood supply, or other non-specific effects. It seemed that information about this might be obtained from the use of an animal model, and we have therefore studied the influence of surgical synovectomy on immune synovitis induced in the rabbit knee joint. This is probably the smallest animal in which such a procedure is practical, and the pattern of immune synovitis in this joint has been extensively studied by Glynn (1968).

The experiment entailed the immunization of rabbits to whole human serum proteins, followed by intra-articular injections of human serum into both knee joints. Later, when there was an active immune synovitis on both sides, surgical synovectomy was performed on one knee, leaving the other joint untouched. Finally, the animals were killed and the state of the two knee joints compared.

Six animals were used in a pilot experiment in order to establish the best anaesthetic and surgical techniques and to study the effect of varying the time between the induction of arthritis, operation, and death. A further ten rabbits were then treated according to the fixed experimental design described below.

\section{Material and Methods}

ANimals

White New Zealand Rabbits weighing between 2.8 and $4.0 \mathrm{~kg}$. were housed in individual wire cages.

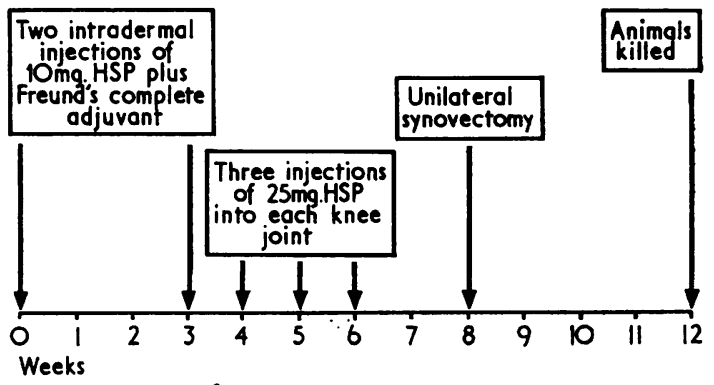

FIG. 1 Design of experiment.

IMMUNIZATION (see Fig. 1)

Sensitizing injections consisted of fresh human serum diluted with physiological saline then emulsified with an equal volume of Freund's complete adjuvant, so that $1 \mathrm{ml}$. emulsion contained $10 \mathrm{mg}$. mixed human serum proteins (HSP). This volume was injected intracutaneously divided between multiple sites on the animal's back, and 3 weeks later the injection was repeated. In the pilot study this regimen was found to produce positive 'delayed' skin tests to $50 \mu \mathrm{g}$. HSP in all of the six animals, and to $10 \mu \mathrm{g}$. in all but one.

INDUCTION OF ARTHRITIS

$25 \mathrm{mg}$. HSP made up to $1 \mathrm{ml}$. with physiological saline was injected into each joint. Strict sterility was observed in the preparation and administration. Eight animals received injections into both knee joints weekly for 3 weeks. Two animals were not injected and served as controls.

\section{ANAESTHESIA}

This was obtained with a 1:50 solution of bromethol (Avertin) given by intraperitoneal injection in a dose of 
$12.5 \mathrm{ml} . / \mathrm{kg}$. body weight. In addition, the periarticular tissues were infiltrated with a solution containing 0.5 per cent. lignocaine and adrenaline 1:10,000.

\section{SYNOVECTOMY}

2 weeks after the last intra-articular injection, surgical synovectomy was carried out on one joint, selected by a system of random allocation. The operation was performed in a manner resembling, as much as possible, that used in the human patient.

After the fur on the leg had been clipped and the skin painted with 1 per cent. iodine in spirit, the area was towelled off, the foot being encased in a glove for convenience.

A lateral, curved, parapatellar incision was made with the knee half flexed, and the capsule opened in line with the incision. A swab was taken for culture, and a synovial biopsy for histology was taken from the lateral condylar area. With the patella dislocated medially, the front of the joint was exposed and all accessible synovium removed, starting with the suprapatellar pouch. The infrapatellar fat pad was excised, together with the synovium around the tendon of extensor digitorum longus. The intercondylar area was also cleared of synovium.

The synovial layer stripped readily when inflamed, but the procedure was difficult in the normal joint. Bleeding was not troublesome and no tourniquet was required.

The capsule was closed with interrupted 3.0 chromic catgut mounted on an atraumatic round-bodied needle, the skin with interrupted 3.0 silk sutures. The wound was sprayed with 'Nobecutane' and a gauze dressing secured with a conforming cotton bandage, which was then saturated with 'Nobecutane'. The dressings and skin sutures were removed after 14 days.

\section{ASSESSMENT OF ARTHRITIS}

Material removed at synovectomy was processed and stained with haemotoxylin and eosin. At the end of the experiment the animals were killed by an intravenous injection of pentobarbitone sodium. A swab for culture was taken from the interior of each knee joint, which was carefully inspected and photographed before removing it complete for decalcification in a formic acid-citrate solution. Blocks were cut sagittally through the knee centre, and additional sections were then made at $90^{\circ}$ to the original cut. All sections were stained with haemotoxylin and eosin, and assessed separately by two observers.

\section{Results}

One animal was found dead in its cage on the day after the operation, leaving seven injected and two non-injected animals for assessment.

After the intra-articular injections all animals developed some swelling of the knee joints. They did not appear distressed, but the caged conditions prevented any assessment of spontaneous activity.

\section{Appearance at synovectomy}

At operation ( 2 weeks after the last intra-articular injection) the appearance ranged from subacute to acute synovitis. The mildest change consisted of hyperaemia of a somewhat thickened synovial membrane and a slight excess of cloudy synovial fluid. The more inflamed joints were distended with semipurulent fluid, and some synovial recesses contained pockets of creamy exudate (always sterile on bacteriological culture). The synovium in these joints was intensely hyperaemic and grossly thickened. In some areas the surface of the articular cartilage looked roughened.

Histologically the synovectomy specimens revealed less variation than was apparent to the naked eye. All the specimens showed an impressive cellular infiltration, mainly of very numerous plasma cells, often in sheets, and moderate numbers of eosinophils. Lymphocytes were present, occasionally forming aggregations, but neutrophil polymorphs were scanty. This exudate sometimes formed a band below the synovial fringe and in other areas was diffusely scattered through oedematous synovial fronds. In addition to hyperaemia and oedema, synovial hyperplasia was invariably present with apparent shedding of superficial cells. Some animals showed small fibrinous exudates. These changes are illustrated in Figs 2 to 4.

\section{Comparison of knee joints at necropsy}

4 weeks after synovectomy when both knee joints were laid open for comparison, the naked eye changes were less marked than those seen at the time of operation. Appearances varied; some joints looked almost normal while others contained a moderate excess of hazy synovial fluid with an obviously thickened pink/grey synovial membrane and roughened articular cartilage. In general, the knees of any one animal showed similar changes; the variation was between different animals rather than between operated and non-operated joints in the same animal. Two operated joints (one injected, one non-injected) had apparently partly subluxated. Apart from this, both the operated and non-operated joints in the control (non-injected) animals appeared normal.

Histological examination revealed changes in nonoperated joints very similar to those seen in the synovectomy specimens: there were, however, more lymphocytes and considerably fewer eosinophils present in the former. On the operated side the synovial cell lining had almost everywhere been restored, although it was rarely more than one cell thick and was based on a more actively fibroblastic subsynovial tissue. There was an inflammatory cell exudate infiltrating the operated side which in no way differed from that on the unoperated side. Most significantly, 'blind' independent scoring of inflammatory features in these sections by two observers showed that, while there was considerable variation from animal to animal, joints on the two sides in 


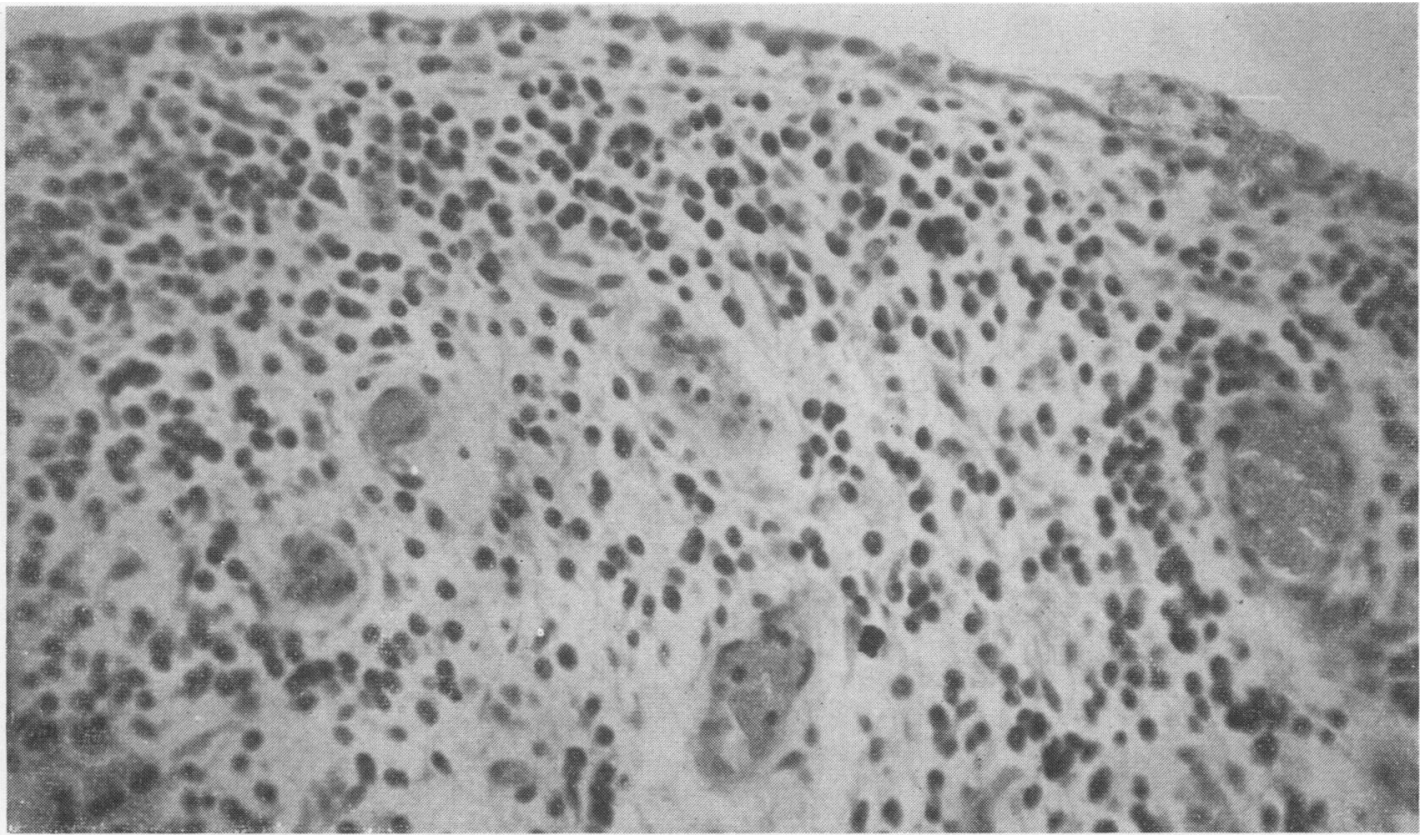

FIG. 2 Synovectomy specimen (Rabbit 4). Moderate infiltration of oedematous stroma by plasma cells and scanty oesinophils. Normal synovial cell layer. Haematoxylin and eosin. $\times 500$.

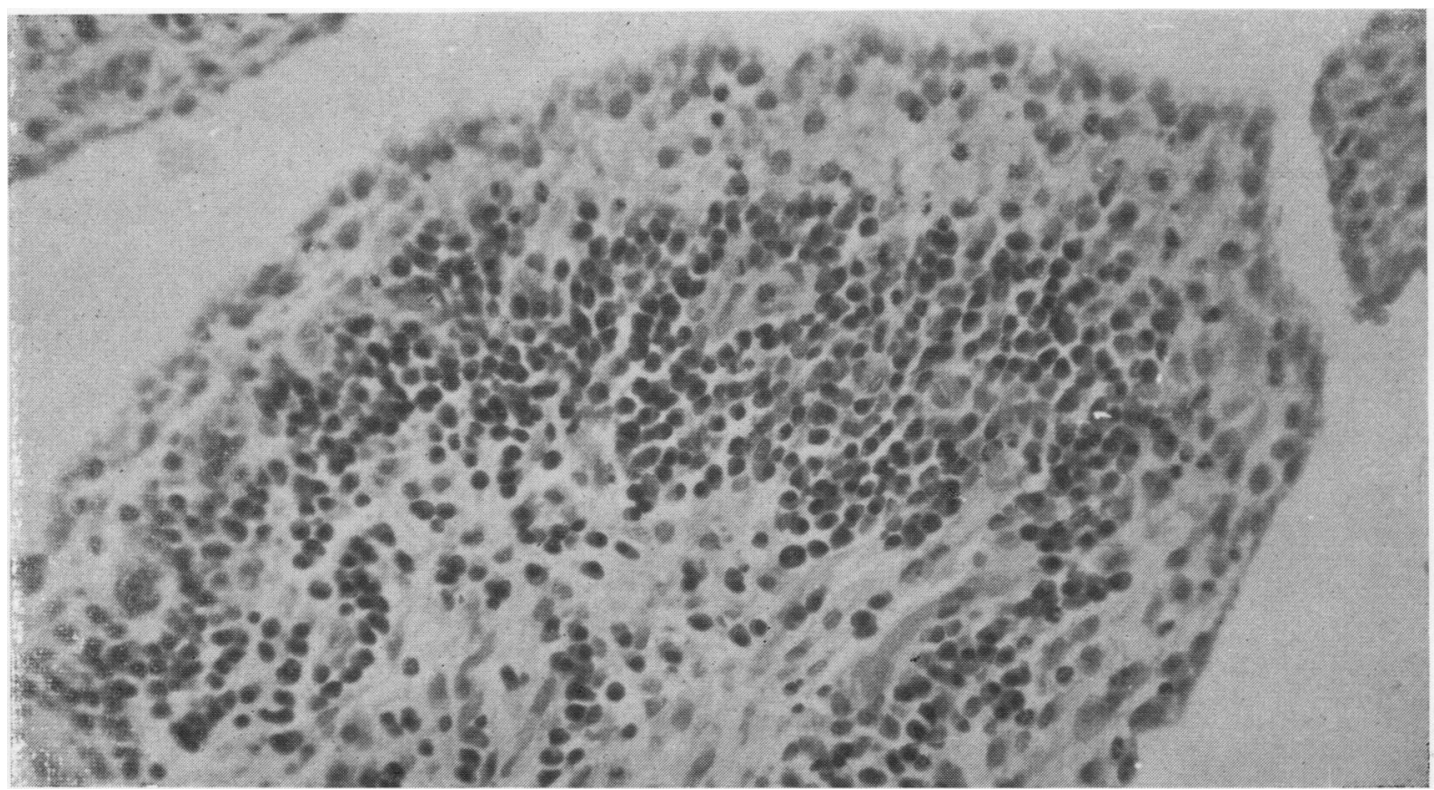

FIG. 3 | Synovectomy specimen (Rabbit, 2 ) $\{$ Broad band of plasma cells, with more superficial eosinophils lying deep to the hyperplastic synovial cell layer. Haemotoxylin and eosin. $\times 500$. 


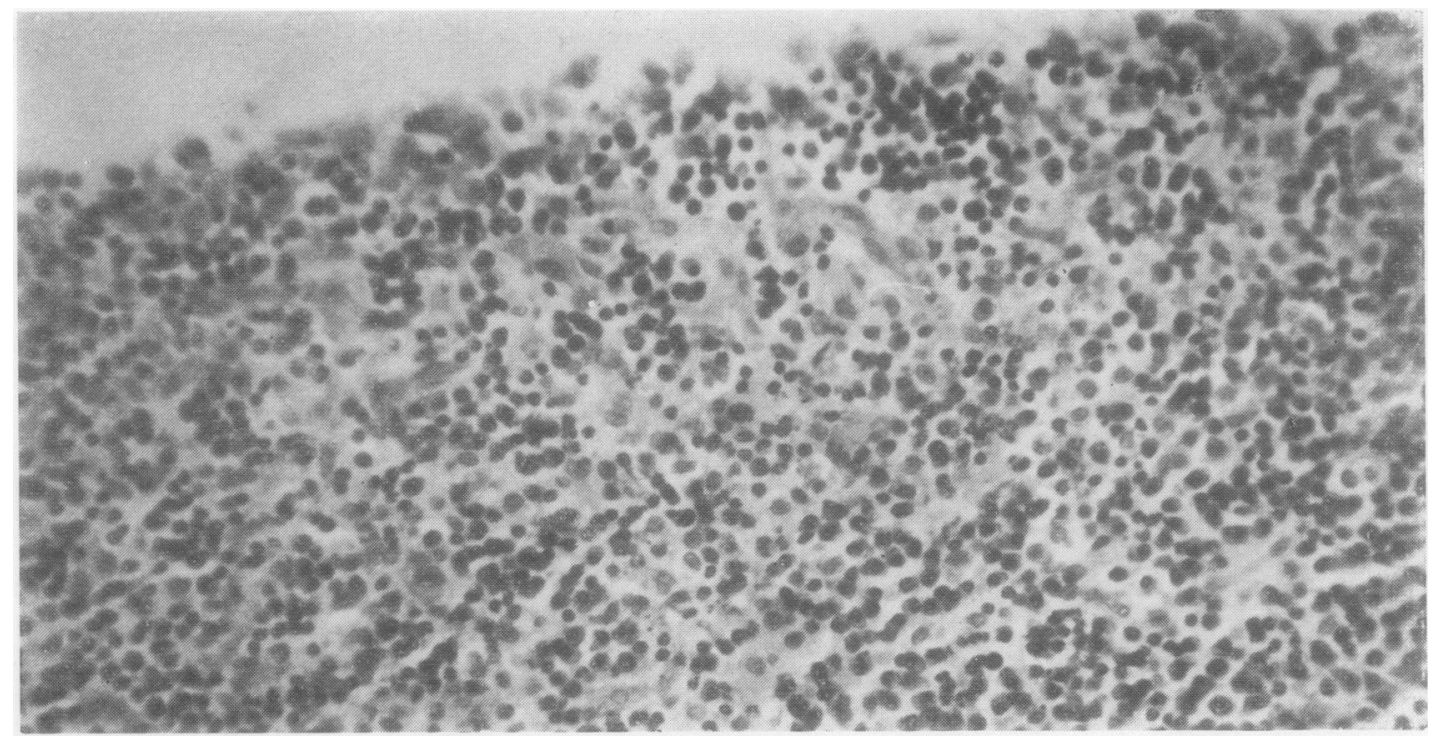

FIG. 4 Synovectomy specimen (Rabbit 8). Very dense infiltration of plasma cells and some eosinophils extending up to synovial cell layer which is hyperplastic and apparently desquamating. Haematoxylin and eosin. $\times 500$.

any particular animal showed essentially comparable injections showed no inflammatory changes. Repreinflammatory changes. Operated joints from the sentative examples of the various appearances two animals which had not received intra-articular described are illustrated in Figs 5 to 7.

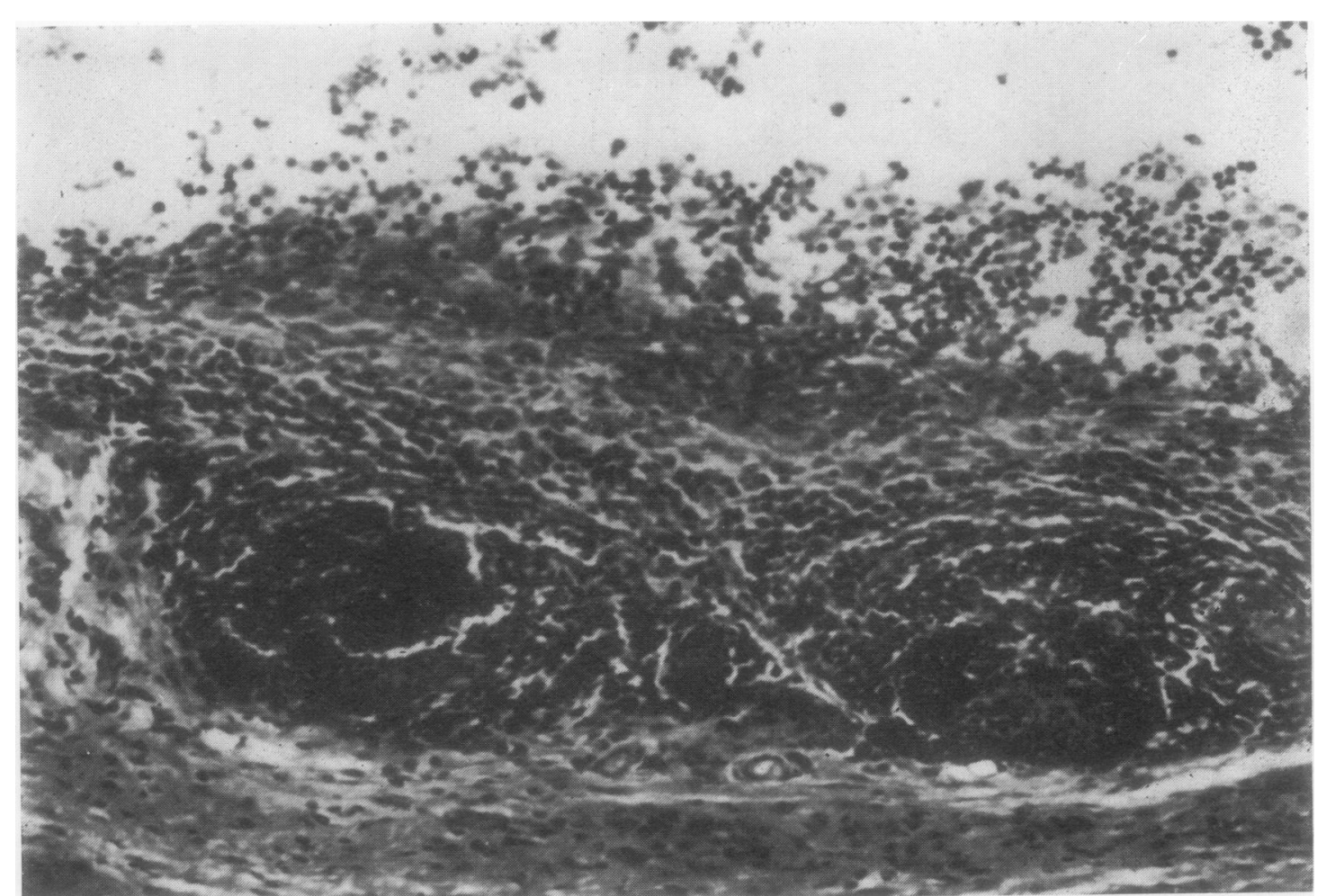

FIG. 5 Unoperated necropsy specimen (Rabbit 7). Two lymphocytic foci surrounded by an infiltration of plasma cells. Synovial layer several cells thick. Sheeding of superficial cells, and inflammatory exudate in joint cavity. Haematoxylin and eosin. $\times 350$. 


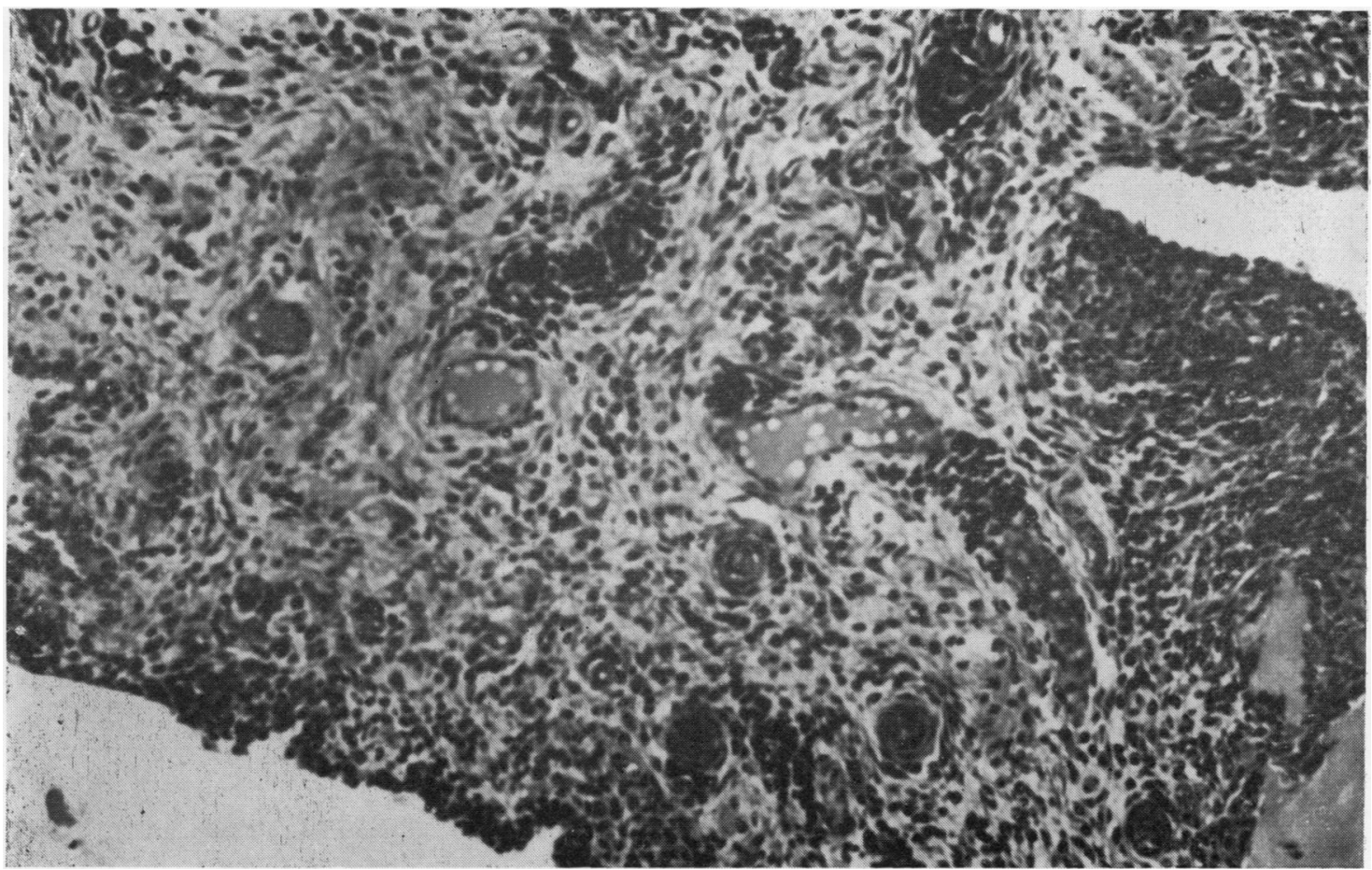

FIG. 6 Typical unoperated necropsy specimen (Rabbit 8). Oedema of stroma, diffuse chronic inflammatory cellular exudate, and some synovial hyperplasia. Haematoxylin and eosin. $\times 350$.

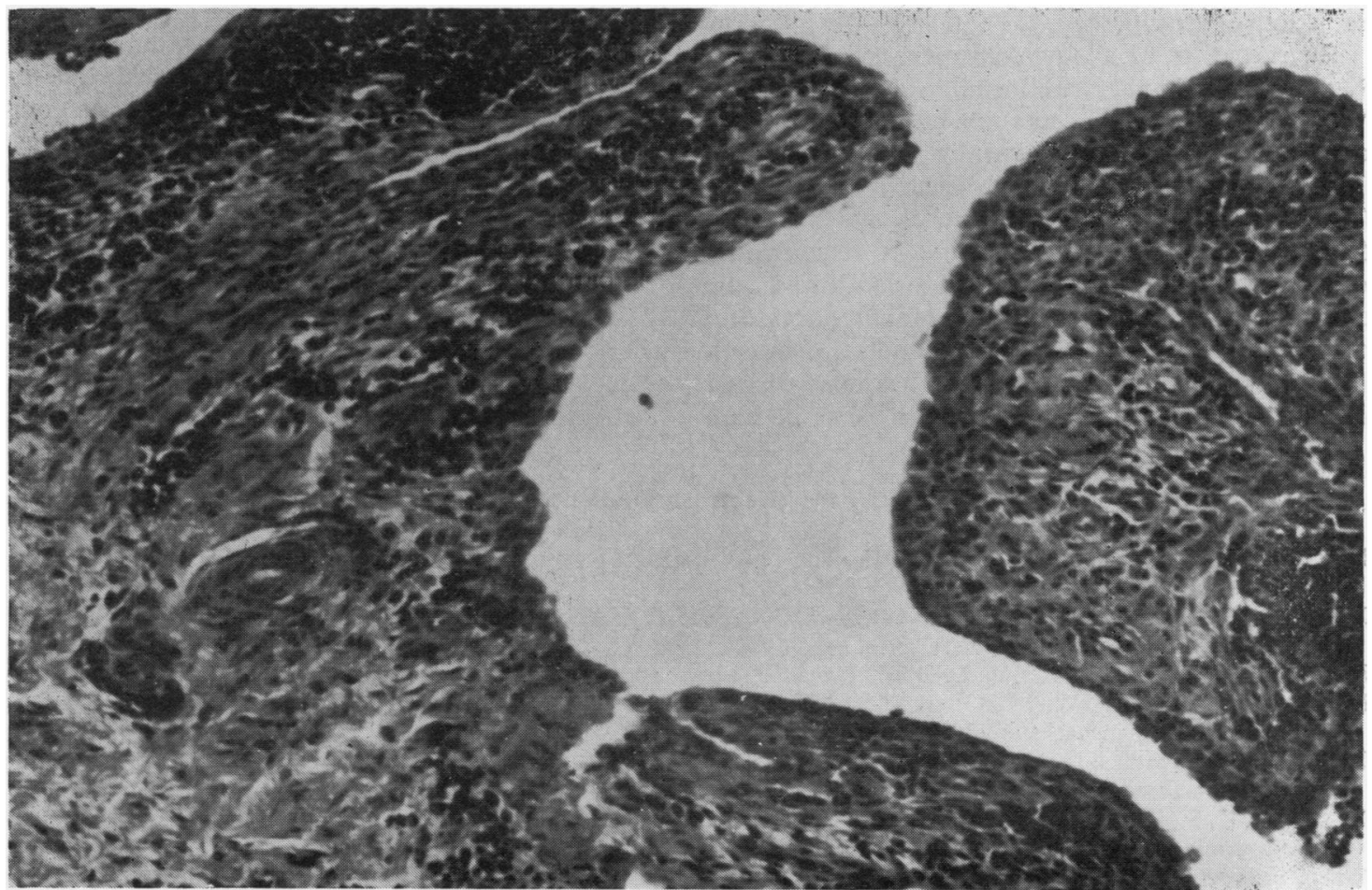

FIG. 7 (Compare with Fig. 6). Typical operated necropsy specimen (Rabbit 2). More collagenous stroma, chronic inflammatory exudate, and regeneration of the synovium mainly as a single layer. Haematoxylin and eosin. $\times 350$. 


\section{Discussion}

This experiment was undertaken in the expectation that synovectomy would modify the course of experimental immune synovitis. This would have provided an experimental approach to such questions as the extent of the excision needed to ensure a beneficial result from this operation. It is not known, for example, whether performing a more complete synovectomy (and thus presumably increasing the risk of subsequent joint contractures) makes it any less likely that the disease will progress locally in that joint.

In fact, these results show that, in this experimental model, synovectomy is followed within 4 weeks by regeneration of the synovial membrane and re-establishment of the original synovitis. Whether this is relevant to the question of what happens in the rheumatoid knee after synovectomy is a matter for speculation. If removal of the responsible antigen is the important factor, then it would be interesting to repeat this experiment using a dose of intra-articular antigen very much smaller than the relatively large doses injected here. In general it does seem that this model holds promise of providing a useful experimental approach to the study of synovectomy. Webb (1969) has already shown that this immune synovitis can be suppressed by measures applicable to the treatment of rheumatoid arthritis. He used intra-articular injections of radioactive yttrium.

Regeneration of synovial membrane after synovectomy in animals is well established (Key, 1925; Wolcott, 1927). This occurs not by the ingrowth of remaining synovial cells, but by the proliferation and metaplasia of local mesenchymal elements (Mitchell and Cruess, 1967). Mitchell and Blackwell (1968), studying the ultrastructure of regenerating rabbit synovial membrane, have followed the development of Type A cells from macrophages, and of Type B cells from fibroblasts. Studies of human knee joints in which synovectomy has been carried out for rheumatoid arthritis confirm that in man also the synovial lining regenerates and not infrequently becomes involved again in the rheumatoid process (Mongan, Boger, Gilliland, and Meyerowitz, 1969; Patzakis, Mills, and Bartholomew, 1969; Ranawat, Straub, Freyberg, Granda, and Rivelis, 1969).

The ideal experimental model for studying surgical synovectomy would be one in which an immune synovitis produced slowly progressive changes over a prolonged period. It should then be possible to test whether the operation modified the advance of the disease on the treated side compared with the opposite joint. In the present experiment the inflammatory process was, if anything, tending to subside during the period between operation and death, so that the situation is not truly analogous to synovectomy carried out in the hope of preventing future exacerbations of rheumatoid arthritis. Nevertheless, the rapid re-establishment of synovitis in the newly regenerated synovium clearly does not provide any experimental support for the concepts on which this operation is based.

\section{Summary}

Rabbits immunized against mixed human serum proteins received intra-articular injections of the same proteins into both knee joints; 2 weeks after the last injection (when an active immune synovitis was in progress), surgical synovectomy was performed on one knee joint in each animal. After a further period of 4 weeks the rabbits were killed and the severity of the arthritis in the two knees was compared. In the operated joints the synovial lining had regenerated and the original type of synovitis had become re-established. There was no evidence that synovectomy had conferred any protection on the operated joints.

It is a pleasure to record our thanks to Dr. R. Pilsworth of the Public Health Laboratory, Chelmsford, who kindly provided animal accommodation for the rabbits used in this study.

\section{References}

GlynN, L. E. (1968) Ann. rheum. Dis., 27, 105 (Heberden Oration: 1967. The chronicity of inflammation and its significance in rheumatoid arthritis).

KeY, J. A. (1925) J. Bone Jt Surg., 7, 793 (The re-formation of synovial membrane in the knees of rabbits after synovectomy).

Mrtchell, N., AND Blackwell, P. (1968). Ibid., 50-A, 675 (The electron microscopy of regenerating synovium after subtotal synovectomy in rabbits).

Mitchell, N. S., AND CRUess, R. L. (1967) Canad. J. Surg., 10, 234 (Synovial regeneration after synovectomy).

Mongan, E. S., Boger, W. M., Gilliland, B. C., AND Meyerowitz, S. (1969) Arthr. and Rheum., 12, 317 (Results of synovectomy in patients with rheumatoid arthritis. A retrospective study).

Patzakis, M. J., Mills, D. M., AND Bartholomew, B. A. (1969) Ibid., 12, 322 (Arthroscopy: A method of evaluating the synovectomized knee).

Ranawat, C. S., Straub, L. R., Freyberg, R., Granda, J. L., and Rivelis, M. (1969) Ibid., 12, 325 (Fate of regenerated synovium following synovectomy of the knee in rheumatoid arthritis. A preliminary report).

WeBB, F. W. S. (1969) Ann. rheum. Dis. 29, 202 (Abstr. Effect of beta particle irradiation upon experimentally-induced chronic inflammatory arthritis).

Wolcotr, W. E. (1927) J. Bone Jt Surg., 9, 67 (Regeneration of the synovial membrane following typical synovectomy). 